\title{
Synthesis, thermal properties, spectroscopic characterization and DFT computations of 1,3-propanediylbis (triphenylphosphonium) peroxydisulfate as a new oxidative agent
}

\author{
Faezeh Makari ${ }^{1^{\dagger}} \quad$ Mostafa Gholizadeh $^{1^{\dagger^{*}}} \quad$ Seyed Reza Nokhbeh ${ }^{1^{\dagger}}$
}

\begin{abstract}
Thermal properties and spectroscopic characterization of new synthesized 1,3-propanediylbis (triphenylphosphonium) peroxydisulfate as a member of phosphonium persulfates were studied. ${ }^{1} \mathrm{H},{ }^{13} \mathrm{C},{ }^{31} \mathrm{P}$ NMR and FT-IR were used for structural characterization of the title salt. To study the thermal behavior of the salt DSC, TG and DTA methods were used. Self-accelerating decomposition temperature of anion determined $200-205^{\circ} \mathrm{C}$ and decomposition point for organic moiety was recorded $340^{\circ} \mathrm{C}$ by DSC method. The specific heat capacity of the salt was determined 1.32 to $1.96 \mathrm{j} / \mathrm{g}{ }^{\circ} \mathrm{C}$ in comparison of sapphire disk between 30 to $160^{\circ} \mathrm{C}$. Theoretical DFT computations such as structural optimization, energy, charge distribution, HOMO-LUMO energy levels and thermochemical parameters were performed with the Gaussian 09 package software using B3LYP/6-31+G* level of theory. Theoretical calculations show HOMO-LUMO energy gap is $3.73 \mathrm{eV}$. Oxidation of several benzylic alcohols was performed by the persulfate salt in the water as a mild oxidative agent under reflux condition.
\end{abstract}

Keywords: thermal properties, phosphonium peroxydisulfate, specific heat capacity, oxidizing agent, Density Function Theory computations

\section{Introduction}

Nowadays, the use of quaternary ammonium, phosphonium and pyridinium salts is a growing trend in various fields of organic synthesis, medicine and industries ${ }^{[1-12]}$. There are many reports on the synthesis of various types of the salts ${ }^{[1,13]}$. Negligible vapor pressure, high thermal stability, appropriate environmental aspects, miscibility with water and polar organic solvents, high viscosity, reusability, their versatility and non-toxicity are some of the interesting properties of these salts. Because of their distinctive properties, these organic salts gained increased interest in the last decade in many fields specifically in organic chemistry as a phase transfer catalyst ${ }^{[14-18]}$, halogenating $^{[19,20]}$, oxidative ${ }^{[21,24]}$, reductive ${ }^{[25]}$ reagents in some organic transformations and as radical initiators in

\footnotetext{
Received: June 22, 2020 Accepted: July 28, 2020 Published: August 5, 2020

* Correspondence to: Mostafa Gholizadeh, Department of chemistry, Faculty of science, Ferdowsi University of Mashhad, Mashhad, I.R., Iran; Email: m_gholizadeh@um.ac.ir

${ }^{\dagger}$ These authors contributed equally to this work and share first authorship

${ }^{1}$ Department of chemistry, Faculty of science, Ferdowsi University of Mashhad, Mashhad, I.R., Iran.

Citation: Makari F, Gholizadeh M and Nokhbeh SR. Synthesis, thermal properties, spectroscopic characterization and DFT computations of 1,3-propanediylbis (triphenylphosphonium) peroxydisulfate as a new oxidative agent. Chem Rep, 2020, 2(1): 144-155.

Copyright: (c) 2020 Mostafa Gholizadeh, et al. This is an open access article distributed under the terms of the Creative Commons Attribution License, which permits unrestricted use, distribution, and reproduction in any medium, provided the original author and source are credited.
}

polymerizations $^{[26]}$.

Many reagents such as chromium trioxide in acetic acid $^{[27,28]}$, ceric ammonium nitrate in aqueous acetic $\operatorname{acid}^{[29]}$, permanganate ion ${ }^{[30]}$ selenium dioxide ${ }^{[31]}$, silver (II) oxide ${ }^{[32]}$, chlorochromate ${ }^{[23]}$ and dichromate ion ${ }^{[21,22]}$ have been used to oxidation of organic substrates. However the above reagents either were used in aqueous acidic solution or in the presence of a metal ion as catalyst. Persulfates are known as oxidizing agents in the preparation of aldehydes, ketones, carboxylic acids, Quinone's and a variety of other compounds. The pharmaceutical industries use sodium persulfate as a reagent in the preparation of antibiotics. Examples of oxidation of organic functional groups with this oxidant ion are: oxidation decarboxylation of acids ${ }^{[32]}$, aromatic hydroxylation $^{[33,34]}$, oxidation of carbon-nitrogen double bonds ${ }^{[35]}$, alkenes $^{[36]}$ and alcohols ${ }^{[37]}$. The reaction of peroxydisulfate ions at ambient temperature is considerably slow therefore, the reaction is accelerated in the presence of $\operatorname{Ag}(\mathrm{I})^{[34]} \mathrm{Cu}(\mathrm{I})^{[34]}$ and $\mathrm{Ni}(\mathrm{II})^{[38,39]}$ under reflux condition. For reactions of alcohols, the oxidation reaction carried out at acidic media and is accompanied by fragmentation $^{[40]}$. Therefore, introduction of a new peroxydisulfate oxidizing agent which works under neutral and aprotic condition for the selective oxidation of alcohols is an important process in organic synthesis ${ }^{[37,41]}$. 
These reagents are comparatively less hazardous, stable, solid, environmentally benign reagents and can be synthesized very easily by precipitating of peroxydisulfate ion with phosphonium, ammonium and pyridinium cations. These salts are sometimes used as a conveniently weighable, green, non-toxic source of oxidizing agents in organic synthesis. Therefore, several oxidizing salts have been synthesized. The most frequently used quaternary phosphonium and ammonium oxidizing reagents are 1,4-bis(triphenylphosphonium) butane peroxydisulfate $^{[42]}, 1$,4-tetrabutylammonium peroxydisulfate ${ }^{[43,45]}$, methyl triphenyl phosphonium peroxydisulfate $^{[46,47]}, 3,6$ bis(triphenylphosphonium)-cyclohexene peroxydisulfate ${ }^{[48]}$.

Sulfate radical anion $\mathrm{SO}_{4}{ }^{\bullet-}$ is one of the most important and powerful oxidant in organic synthesis ${ }^{[49]}$. The first step of oxidation of organic functional groups by peroxydisulfate anion is the decomposition of the anion which has a relatively high oxidation potential and an activation energy of approximately $30 \mathrm{Kcal} / \mathrm{mol}^{[44]}$.

$$
\begin{gathered}
S_{2} O_{8}^{2-}{ }_{(a q)}+2 e \rightarrow 2 S_{4}^{2-}{ }_{(a q)} \quad E_{O}=2.01 \mathrm{~V} \\
S_{2} O_{8}^{2-} \rightarrow 2 S_{4}^{\bullet-} \quad E_{a c t}=30 \mathrm{Kcal} / \mathrm{mol}
\end{gathered}
$$

Also, decomposition of the anion is accelerated by strong mineral acids or by trace metal ions ${ }^{[50]}$. The readily available peroxydisulfate salt is an excellent and versatile oxidant for most of organic compounds.

Thus, in this article we report the synthesis, thermal properties, structural characterization $\left({ }^{1} \mathrm{H},{ }^{13} \mathrm{C},{ }^{31} \mathrm{P} \mathrm{NMR}\right.$, FT-IR, and DSC/TG/DTA) and DFT calculations of 1,3propanediylbis (triphenylphosphonium) peroxydisulfate as a mild, inexpensive and efficient oxidizing agent. Furthermore, we obtained specific heat capacity of the new salt and related bromide salt by DSC method. We also report the use of new salt to oxidation of benzylic alcohols in water as an environmental protections, clean and green solvent with excellent yields.

\section{Results and discussion}

1,3-Propanediylbis(triphenylphosphonium) dibromide I, prepared initially as white powder by the reaction of triphenyl phosphine with 1,3-dibromopropane (m.p.: 349$354^{\circ} \mathrm{C}$ by DSC method, $95 \%$ yield $)^{[51]}$ and then treated with an aqueous solution of ammonium peroxydisulfate in the ratio 1:1, white precipitate II was filtered and dried under a stream of air (Figure 1) (self-accelerating decomposition temperature SADT $200-205^{\circ} \mathrm{C}$ by DSC method, 97\% yield) Figure 2.

\subsection{Thermal properties of salt II}

DSC thermogram shows a sharp and strong exothermic peak at the $200-205^{\circ} \mathrm{C}$ as a decomposition point of

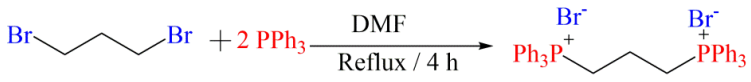

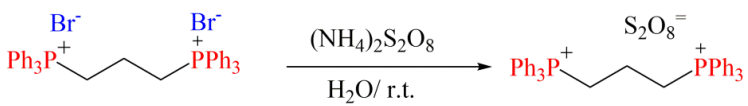

Figure 1. Schematic reactions for preparation of 1,3-propanediylbis (triphenylphosphonium) bromide I and peroxydisulfate II

persulfate ion (self-accelerating decomposition temperature SADT) according to following reaction and a weak endothermic broad peak at the $320-360^{\circ} \mathrm{C}$ as a decomposition range for the organic moiety of compound II. Figure 2. DSC and TG/DTG/DTA thermograms for compound I are reported in our previous work ${ }^{[51]}$.

$$
\mathrm{S}_{2} \mathrm{O}_{8}^{2-}+\text { heat } \rightarrow 2 \mathrm{SO}_{4}^{\bullet-}
$$

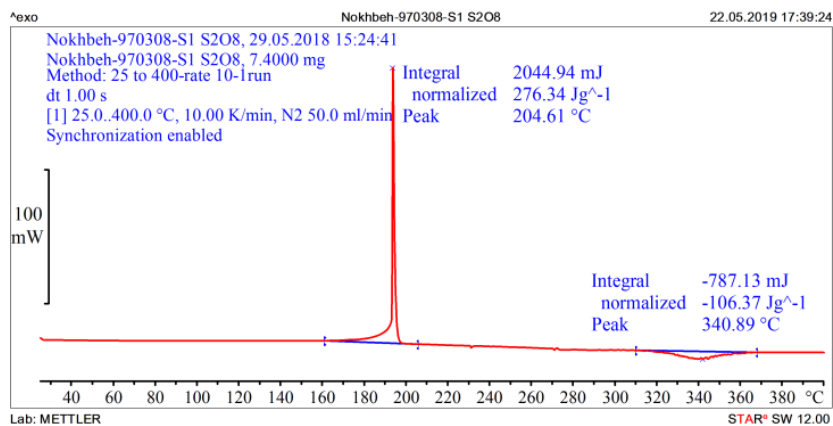

Figure 2. DSC thermogram of compound II

In the TG/DTG/DTA thermogram of II we can see an exothermic strong and sharp peak at about $204^{\circ} \mathrm{C}$ in DTA (blue) curve and a small decrease in TG (red) curve $(4.1 \%)$ for decomposition of peroxydisulfate anion. The small broad endothermic peak in the range of $310-350^{\circ} \mathrm{C}$ in the DTA curve and strong, broad peak in DTG (brown) curve with a significant sharp decrease in TG (red) curve $(90.2 \%)$ for the complete decomposition of compound II and about $5.6 \%$ at $800^{\circ} \mathrm{C}$ for ash was observable which are completely matched with DSC thermograms (Figure 3). Comparison of DSC and TG/DTG/DTA thermograms shows that compound $\mathbf{I}$ is more thermally stable than compound II. Because the structure of the cation is the same in both compounds, I and II, so the thermal instability of the II can be attributed to the difference in anions. For compound II, before it reaches the melting point suffer from accelerating decomposition phenomenon. In addition, the auto oxidation of II by anion can be considered. Anyway, both salts are very stable on the bench-top in the form of powder or in the organic solvent and in contact with air without any destruction over 9 months. In comparison with potassium, ammonium and sodium peroxydisulfate as common inorganic persulfates (decomposition point $<$ 


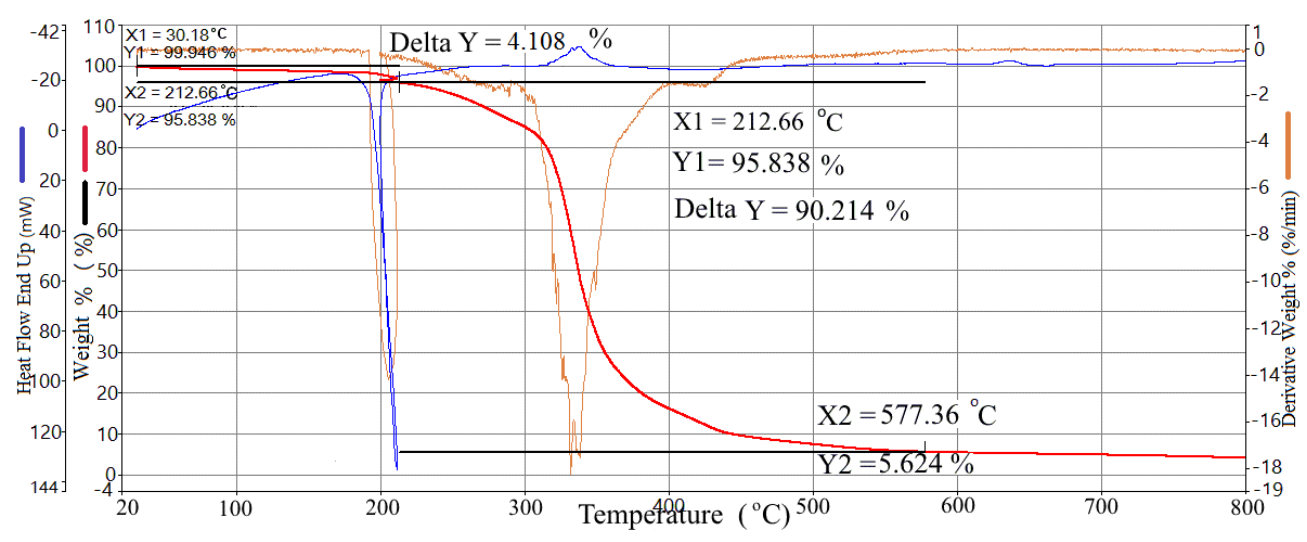

Figure 3. TG/ DTG/ DTA thermogram of compound II

$100,120,180^{\circ} \mathrm{C}$, respectively) that are used as oxidative reagents, compound II has a higher decomposition point and more thermal stability.

Heat capacity is one of the most important physical and thermal properties of materials. To understand the properties of materials and their reactions, it is significant to have deep knowledge of thermodynamic functions such as enthalpy, entropy, and Gibbs free energy. These functions can be directly determined from the specific heat capacity. It is a key property for designing of chemical processes such as injection molding, crystallization, spray drying, the safety analysis of chemical processes and the design of chemical reactors (process engineering) that can be measured by DSC method. In literature, it is defined as the amount of thermal energy required to raise the temperature of an amount of a substance per unit of mass. It is an extensive property since its value is directly proportional to the mass of the substance. The heat capacity of an object, defined by $\mathrm{C}$ is:

$$
C=\lim _{\Delta T \rightarrow 0} \frac{\Delta Q}{\Delta T}
$$

Where $\Delta \mathrm{Q}$ is the amount of heat that must be given to the mater (of mass $M$ ) to raise its temperature by $\Delta \mathrm{T}$. The value of this parameter usually depending on the starting temperature $\mathrm{T}$ of the object and the pressure $\mathrm{P}$ applied to it. Therefore, it should be considered as a function of those two variables $\mathrm{C}(\mathrm{P}, \mathrm{T})$. The SI unit for heat capacity of an object is a joule per Kelvin $\left(\mathrm{J} / \mathrm{K}\right.$, or $\left.\mathrm{J} \mathrm{K}^{-1}\right)$.

A more meaningful material property is the specific heat capacity, $c_{p}$, with a small $\mathrm{c}$ and the subscript $p$. The specific heat capacity is the amount of heat energy that required to raising the temperature of $1 \mathrm{~kg}$ of the substance by $1^{\circ} \mathrm{C}$ or $1 \mathrm{~K}$. The SI unit for specific heat is joules per Kelvin per kilogram $\left(\mathrm{J} / \mathrm{K} / \mathrm{kg}, \mathrm{J} /(\mathrm{kg} \mathrm{K}), \mathrm{J} \mathrm{K}^{-1} \mathrm{~kg}^{-1}\right.$, etc.) For specific heat capacity, we can write:

$$
C_{P}=\frac{d Q}{d T} \cdot \frac{1}{m}
$$

These definitions were only valid in the absence of chemical reactions and/or phase transitions. Nowadays, this concept has been expanded. In the absence of phase transitions and chemical reactions, $c_{p}$ is referred to as the baseline $c_{p}$. The deviation caused by phase transitions and chemical reactions is valid the excess $c_{p}$ and the sum of both is known as the total specific heat capacity. If we divide the heat capacity by the mole number, we get the molar heat capacity in units of Joules per mole Kelvin.

Thus, we determined and reported specific heat capacity of $\mathbf{I}$ and II by DSC method in the range of 30 to $160^{\circ} \mathrm{C}$ in comparison of sapphire disk and with automatic blank curve subtraction (Figure 4 and Figure 5 ). A comparative measurement was frequently used which involved measuring a reference material (sapphire, single crystal alumina $\alpha-\mathrm{Al}_{2} \mathrm{O}_{3}$ ) under the same conditions as the sample. A simple calculation, then allowed the unknown specific heat capacity of the sample to be obtained from the known specific heat capacity of $c_{p s}$ of $\alpha-\mathrm{Al}_{2} \mathrm{O}_{3}$. Numerical data are tabulated in Table 1.

$$
C_{p}=\frac{\Phi}{\Phi_{S}} \cdot \frac{m_{S}}{m} C_{p s}
$$

Comparison of plots for two compounds shows that the specific heat capacity is between 0.79 to $1.26 \mathrm{j} / \mathrm{g}{ }^{\circ} \mathrm{C}$ for I and in the range of 1.32 to $1.96 \mathrm{j} / \mathrm{g}{ }^{\circ} \mathrm{C}$ for II and that the specific heat capacity of II is higher than I in a whole range (Figure 6, Table 1).

\subsection{Theoretical DFT computation}

Over the recent year's computational calculation based on the DFT theory has been developed to investigate and predict the structure, vibrational, magnetic and electrical, thermal and spectroscopic properties of substances ${ }^{[52-59]}$. The Gaussian 09 software package was used to perform the DFT calculations at the B3LYP (Becke-3- Lee-YangPar) level and with 6-311+G* basis sets for compound $\mathbf{I}$ and II. The DFT computations were done by using the 


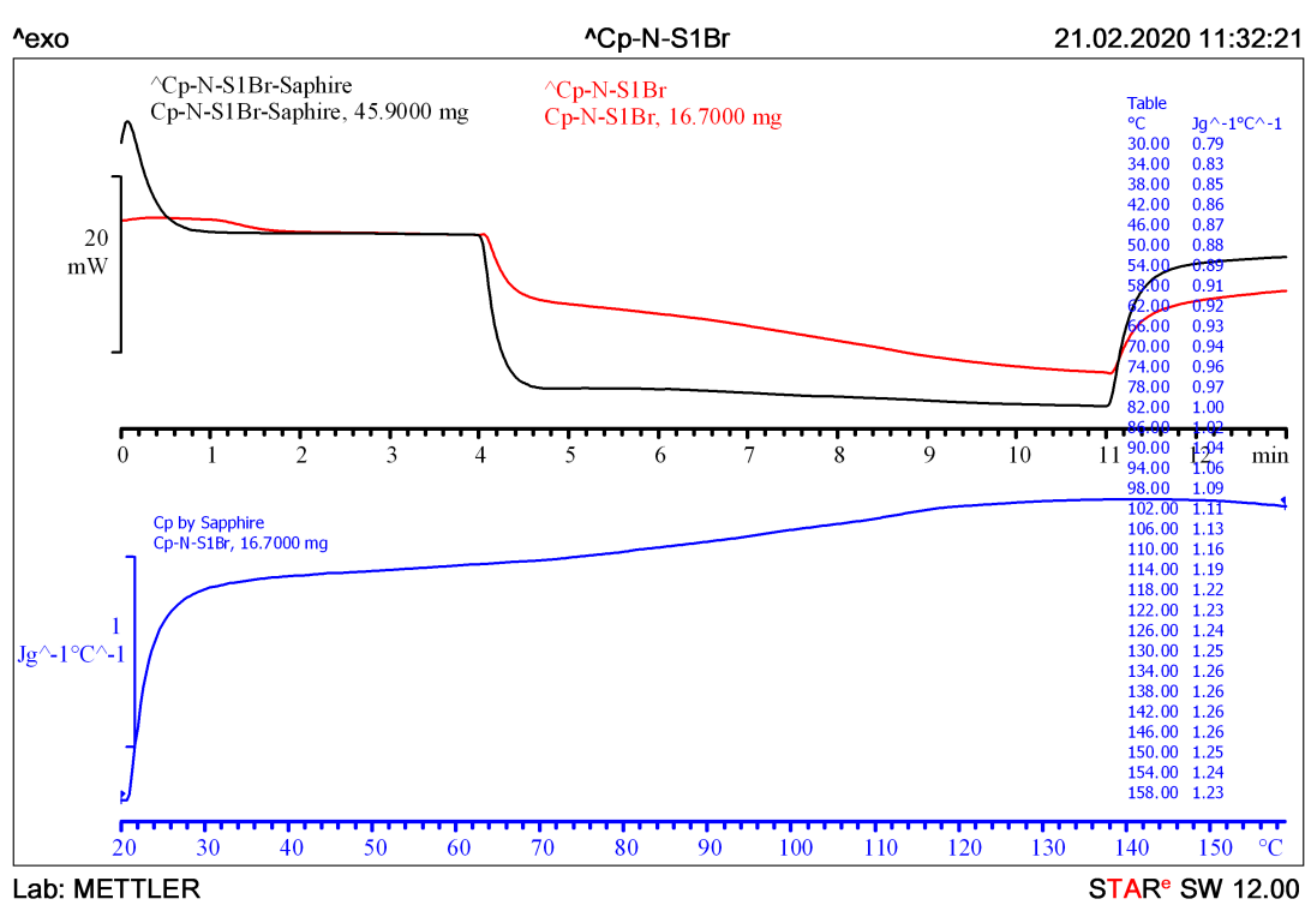

Figure 4. Plot of specific heat capacity for I

Gaussian 09 software package at the B3LYP/6-31+G* basis sets for title compound II. The structural parameters such as bond lengths, bond angles and dihedral (torsion) angles thermochemical parameters and were computed after optimization of structure (Table 2 and Table 3 ). The DFT calculation predicted energy and dipole moment for I, E (RB3LYP) -7334.05720641 Hartrees, Dipole moment $=16.9062$ Debye $^{[51]}$, while for II, E (RB3LYP) $=$ -3589.09159282 Hartrees, Dipole moment 25.0194 Debye.

B3LYP/6-31+G* method was used to calculate 255 vibrational frequencies for compound II. Highest predicted frequencies are $3147.96 \mathrm{~cm}^{-1}$ for the stretching vibration of $\mathrm{C}-\mathrm{H}$ in aromatic rings, $3048.66 \mathrm{~cm}^{-1}$ for the in plane symmetric stretching of aliphatic H-C-H, $2987.09 \mathrm{~cm}^{-1}$ for the stretching vibration of aliphatic C-H, 1441.19 $\mathrm{cm}^{-1}$ for the stretching vibration of $\mathrm{S}=\mathrm{O}, 1357.74 \mathrm{~cm}^{-1}$ for the asymmetric stretching of $\mathrm{O}=\mathrm{S}=\mathrm{O}$ and $772.81 \mathrm{~cm}^{-1}$ for the stretching of S-O in anion (Table 4).

The results extracted from NBO calculations have provided deeper insight into the nature of electronic structure of molecule $\mathbf{I I}^{[60]}$. Since atomic charges affect some properties of molecular systems, including electronic structure, dipole moment, molecular polarizability; atomic charges calculation has an important role in the quantum chemical calculation of molecular systems. NBO method was used to calculate natural charge distribution on the whole molecule of II. Calculation show the amount of charge distribution on atoms and indicated that $\mathrm{C}$ and $\mathrm{O}$ atoms have negative character; while $\mathrm{P}, \mathrm{S}$ and $\mathrm{H}$ atoms have positive ones (Table 5 ).

HOMO and LUMO orbitals are referred to frontier orbitals and the difference in energy between these orbitals is termed HOMO-LUMO gap that can be used to predict chemical stability and reactivity of organic compounds ${ }^{[61,62]}$. The energy level of frontier orbitals shows a tendency to giving an electron as a donor or to take an electron as an acceptor in chemical reactions. HOMOLUMO energies were calculated by B3LYP/6-31+G* presented in Figure 7. In comparison with $\mathbf{I}$; although the HOMO and LUMO energy levels have been reduced, but the HOMO level is dropped significantly and as a result the energy gap increased from $2.38 \mathrm{eV}$ (for I) to $3.73 \mathrm{eV}$ (for II). This means that although the compound II has become more stable than I with anion exchange, in the red-ox reactions it plays the role of an electron acceptor.

\section{Materials and methods}

All chemicals, including raw materials, reagents and solvents were purchased from Merck Co. 1,3dibromopropane was purified and checked by Gas Chromatograph to give purity not less than $99 \%$. The progresses of reactions were monitored by TLC and GC. ${ }^{1} \mathrm{H}$, ${ }^{13} \mathrm{C}$ and ${ }^{31} \mathrm{P}$ NMR spectra were recorded by the Bruker AC 300 and $500 \mathrm{MHz}$ spectrometer with $\mathrm{D}_{2} \mathrm{O}, \mathrm{CDCl}_{3}$ and DMSO- $d_{6}$ as solvents. $c_{p}$ and DSC thermograms were recorded by DSC-822e METTLER -TOLEDO thermal 


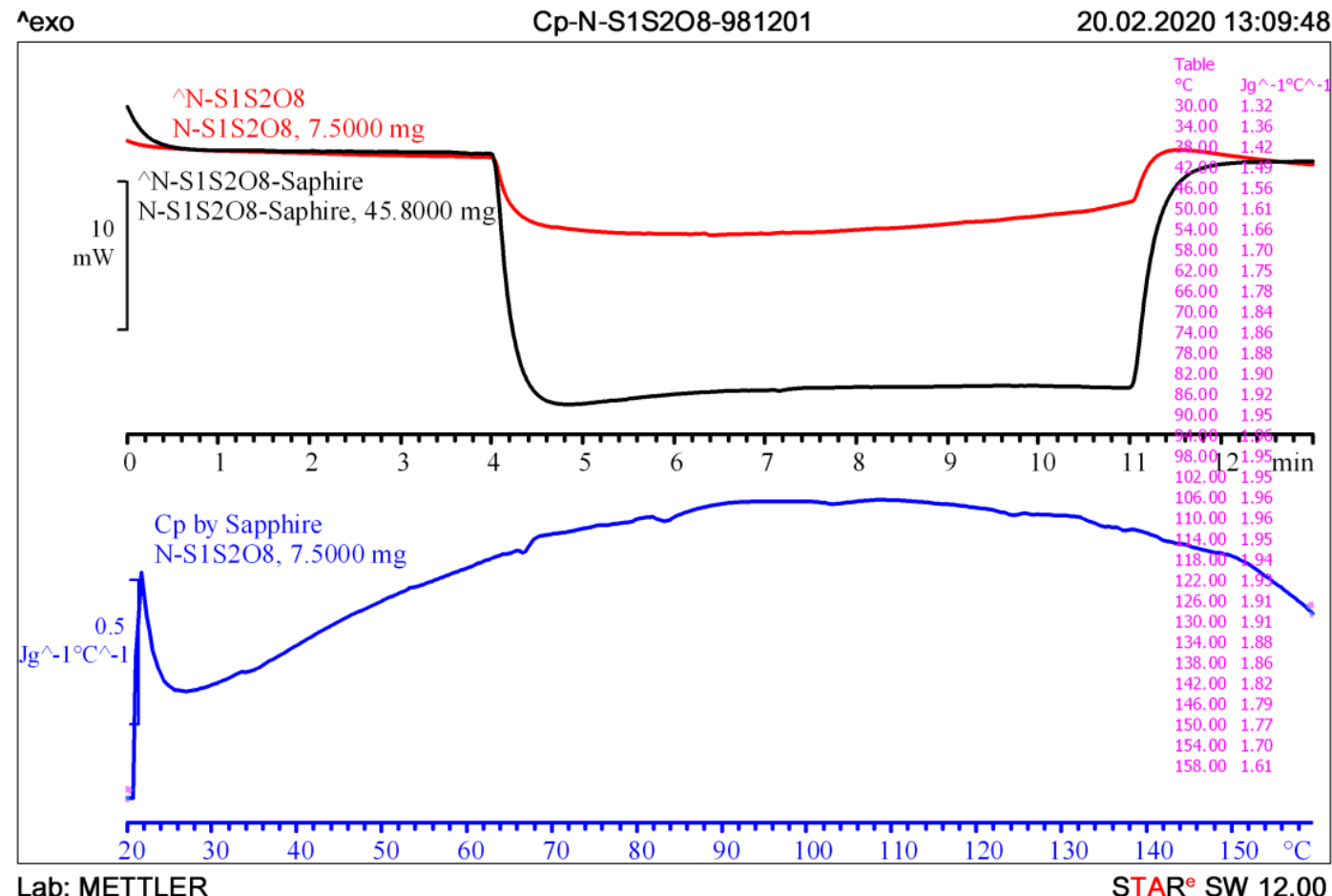

Figure 5. Plot of specific heat capacity for II

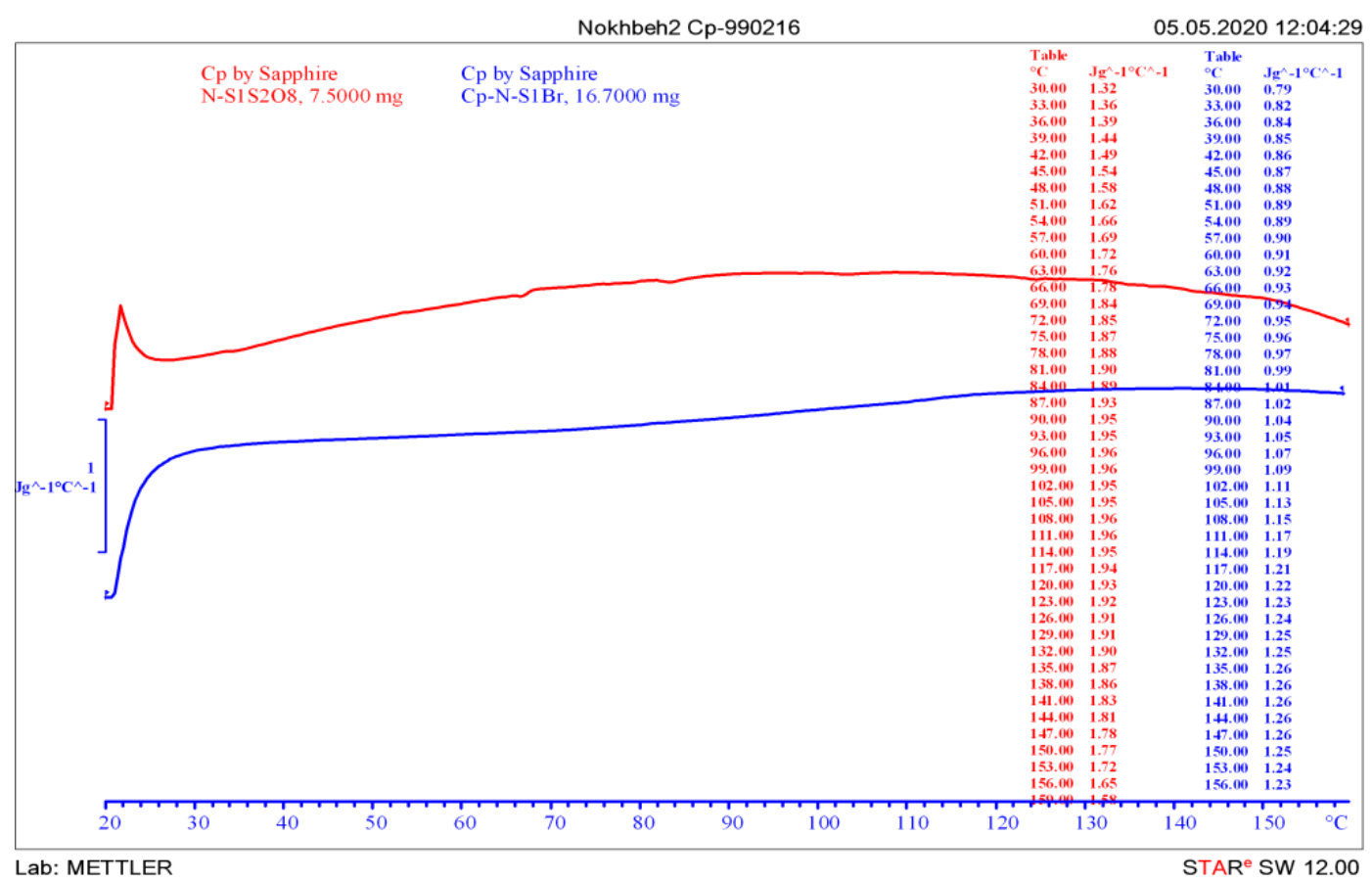

Figure 6. Comparison of specific heat capacity of I and II 
Table 1. Numerical data for specific heat capacity $\mathrm{C}_{p}$ of I \& II

\begin{tabular}{ccc|ccc}
\hline $\begin{array}{c}\text { Temp } \\
{ }^{\circ} \mathrm{C}\end{array}$ & $\begin{array}{c}\mathrm{I} \\
\mathrm{J} / \mathrm{g} /{ }^{\circ} \mathrm{C}\end{array}$ & \begin{tabular}{c}
$\mathrm{II} / \mathrm{g} /{ }^{\circ} \mathrm{C}$ \\
\hline 30
\end{tabular} & $\begin{array}{c}\text { Temp } \\
{ }^{\circ} \mathrm{C}\end{array}$ & $\begin{array}{c}\mathrm{I} \\
\mathrm{J} / \mathrm{g} /{ }^{\circ} \mathrm{C}\end{array}$ & $\begin{array}{c}\mathrm{II} \\
\mathrm{J} / \mathrm{g} /{ }^{\circ} \mathrm{C}\end{array}$ \\
\hline 32 & 0.81 & 1.32 & 96 & 1.07 & 1.96 \\
34 & 0.83 & 1.36 & 98 & 1.09 & 1.95 \\
36 & 0.84 & 1.39 & 102 & 1.1 & 1.96 \\
38 & 0.85 & 1.42 & 104 & 1.12 & 1.95 \\
40 & 0.86 & 1.46 & 106 & 1.13 & 1.95 \\
42 & 0.86 & 1.49 & 108 & 1.15 & 1.96 \\
44 & 0.87 & 1.52 & 110 & 1.16 & 1.96 \\
46 & 0.87 & 1.56 & 112 & 1.17 & 1.96 \\
48 & 0.88 & 1.58 & 114 & 1.19 & 1.95 \\
50 & 0.88 & 1.61 & 116 & 1.2 & 1.95 \\
52 & 0.89 & 1.64 & 118 & 1.22 & 1.94 \\
54 & 0.89 & 1.66 & 120 & 1.22 & 1.93 \\
56 & 0.9 & 1.68 & 122 & 1.23 & 1.93 \\
58 & 0.91 & 1.7 & 124 & 1.23 & 1.91 \\
60 & 0.91 & 1.72 & 126 & 1.24 & 1.91 \\
62 & 0.92 & 1.75 & 128 & 1.25 & 1.91 \\
64 & 0.92 & 1.77 & 130 & 1.25 & 1.91 \\
66 & 0.93 & 1.78 & 132 & 1.25 & 1.9 \\
68 & 0.93 & 1.83 & 134 & 1.26 & 1.88 \\
70 & 0.94 & 1.84 & 136 & 1.26 & 1.87 \\
72 & 0.95 & 1.85 & 138 & 1.26 & 1.86 \\
74 & 0.96 & 1.86 & 140 & 1.26 & 1.85 \\
76 & 0.97 & 1.87 & 142 & 1.26 & 1.82 \\
78 & 0.97 & 1.88 & 144 & 1.26 & 1.81 \\
80 & 0.98 & 1.89 & 146 & 1.26 & 1.79 \\
82 & 1 & 1.9 & 148 & 1.26 & 1.78 \\
84 & 1.01 & 1.89 & 150 & 1.25 & 1.77 \\
86 & 1.02 & 1.92 & 152 & 1.25 & 1.74 \\
88 & 1.03 & 1.94 & 154 & 1.24 & 1.7 \\
90 & 1.04 & 1.95 & 156 & 1.23 & 1.65 \\
92 & 1.05 & 1.95 & 158 & 1.23 & 1.61 \\
94 & 1.06 & 1.96 & & & \\
\hline & & & & &
\end{tabular}

analyzer. TG/DTG/DTA thermograms were recorded by Perkin Elmer Diamond TG/DTA Instrument. Infrared spectra were recorded on a Perkin Elmer-Spectrum 65FT-IR and Bruker vortex 80 spectrometers as a KBr disk (400 - $4000 \mathrm{~cm}^{-1}$ region).

\subsection{Preparation of 1,3-propanediylbis bro- mide $\left(\mathbf{C}_{39} \mathbf{H}_{36} \mathbf{P}_{2} \mathrm{Br}_{2}\right)$ I}

Triphenylphosphine (6.55 g, $25 \mathrm{mmol}$, excess) was dissolved in DMF $(30 \mathrm{~mL})$ in a $50 \mathrm{~mL}$ roundbottom flask and magnetically stirred under reflux condition. 1,3Dibromopropane (2.01 g, $1.01 \mathrm{~mL}, 10 \mathrm{mmol}, \mathrm{d}=1.989$ $\mathrm{g} / \mathrm{cm}^{3}$ ) was added dropwise with a $1 \mathrm{~mL}$ syringe. The mixture was refluxed for over $4 \mathrm{~h}$. The white precipitate was cooled, filtered and washed with DMF $(3 \times 10 \mathrm{~mL})$. The product was air dried overnight (6.9 g, 95\% yield, purity after recrystallization in hot water 99.7\%), m.p. $349-353^{\circ} \mathrm{C}$ (by DSC and DTA) ${ }^{[51]}$.

\subsection{Preparation of 1,3-propanediylbis perox- ydisulfate $\left(\mathrm{C}_{39} \mathbf{H}_{36}-\mathbf{P}_{2} \mathbf{S}_{2} \mathbf{O}_{8}\right)$ II}

$2.38 \mathrm{~g}$, (20 mmol) of compound I was dissolved in $\mathrm{H}_{2} \mathrm{O}$ $(30 \mathrm{~mL})$ at $70^{\circ} \mathrm{C}$ in a $50 \mathrm{~mL}$ beaker, an aqueous solution of ammonium peroxydisulfate $(1.03 \mathrm{~g}, 20 \mathrm{mmol}$ in $50 \mathrm{~mL}$
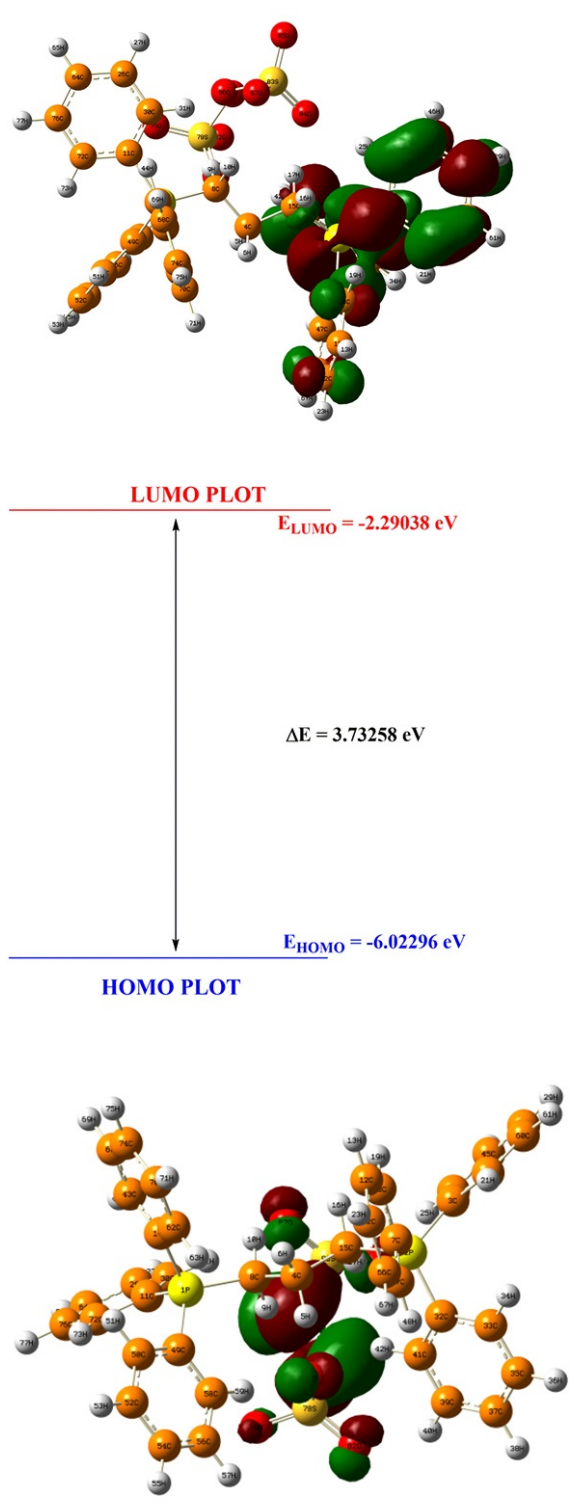

Figure 7. HOMO-LUMO diagram and energy levels for II

water) was added dropwise with continuous magnetically stirring for $30 \mathrm{~min}$. Solution was mixed for over $20 \mathrm{~min}$. The white precipitate was filtered and washed with cooled water $(3 \times 10 \mathrm{~mL})$. The product was air dried overnight and crystallized in $\mathrm{H}_{2} \mathrm{O} / \mathrm{EtOH} 50 \% \mathrm{~V} / \mathrm{V}$ (97\% yield, purity after recrystallization $99.5 \%$ ), decomposition point $340^{\circ} \mathrm{C}$ (by DSC and DTA). ${ }^{1} \mathrm{H}$ NMR $(300 \mathrm{MHz}$, DMSO$d_{6}, \delta$; ppm): 1.80 ppm (s, $2 \mathrm{H}$, center $\left.\mathrm{CH}_{2}\right), 3.88$ (s, 4H, terminal $\mathrm{CH}_{2}$ 's), 7.76-7.91 (m, 30H, aromatic hydrogens). ${ }_{31}$ P NMR (200MHz, DMSO-d6, ; ppm): 34.74 ppm (1 $\mathrm{s}$, for $\mathrm{P}$ atom). ${ }^{13} \mathrm{C}$ NMR (75 MHz, DMSO- $d_{6}, \delta$; ppm): $16.6\left(\mathrm{C}_{2} 0\right), 20.72$ (C21, C19), 117.81, 118.96, 130.80, 134.15 and $135.56\left(5 \times \mathrm{s}, \mathrm{C}_{6} \mathrm{H}_{5}\right)$. FT-IR: $\bar{\nu}=509,539$ (m), 686,723 (m), 996 (w), 1040 (s), 1112 (s), 1249, 1267 (s), 1439 (m), 1486, 1589 (w), 2904, 2938 (w), 3060 (w) 
Table 2. Cartesian coordinates for compound II

\begin{tabular}{|c|c|c|c|c|c|c|c|c|c|c|c|}
\hline \multirow{2}{*}{$\begin{array}{l}\text { Center } \\
\text { Number }\end{array}$} & \multirow{2}{*}{$\begin{array}{l}\text { Atomic } \\
\text { Number }\end{array}$} & \multirow{2}{*}{$\begin{array}{l}\text { Atomic } \\
\text { Type }\end{array}$} & \multicolumn{3}{|c|}{ Coordinates } & \multirow{2}{*}{$\begin{array}{l}\text { Center } \\
\text { Number }\end{array}$} & \multirow{2}{*}{$\begin{array}{l}\text { Atomic } \\
\text { Number }\end{array}$} & \multirow{2}{*}{$\begin{array}{l}\text { Atomic } \\
\text { Type }\end{array}$} & \multicolumn{3}{|c|}{ Coordinates } \\
\hline & & & $X$ & Y & $\mathrm{Z}$ & & & & $X$ & $\mathrm{Y}$ & $\mathrm{Z}$ \\
\hline 1 & 15 & 0 & -2.7286 & 0.981156 & -0.16078 & 45 & 6 & 0 & 4.890518 & -1.21773 & -3.15419 \\
\hline 2 & 15 & 0 & 3.030109 & 0.615363 & -0.01612 & 46 & 1 & 0 & 4.697306 & -2.08425 & -3.7801 \\
\hline 3 & 6 & 0 & 4.206602 & 0.248681 & -1.35562 & 47 & 6 & 0 & 3.205255 & 2.767115 & 1.790462 \\
\hline 4 & 6 & 0 & 0.14858 & 0.904342 & 0.077057 & 48 & 1 & 0 & 3.213644 & 2.018086 & 2.576188 \\
\hline 5 & 1 & 0 & 0.213581 & 0.706817 & 1.151151 & 49 & 6 & 0 & -2.99729 & 1.305632 & 1.605887 \\
\hline 6 & 1 & 0 & 0.124786 & 1.991171 & -0.06464 & 50 & 6 & 0 & -3.75463 & 2.409437 & 2.037789 \\
\hline 7 & 6 & 0 & 3.171342 & 2.37552 & 0.443582 & 51 & 1 & 0 & -4.13546 & 3.134108 & 1.323974 \\
\hline 8 & 6 & 0 & -1.10948 & 0.20963 & -0.48208 & 52 & 6 & 0 & -4.02083 & 2.578929 & 3.396994 \\
\hline 9 & 1 & 0 & -1.15225 & -0.80736 & -0.07095 & 53 & 1 & 0 & -4.60683 & 3.432279 & 3.727942 \\
\hline 10 & 1 & 0 & -1.04819 & 0.101192 & -1.57197 & 54 & 6 & 0 & -3.53827 & 1.650883 & 4.325807 \\
\hline 11 & 6 & 0 & -4.01951 & -0.13459 & -0.77788 & 55 & 1 & 0 & -3.75064 & 1.783255 & 5.383634 \\
\hline 12 & 6 & 0 & 3.176151 & 4.709933 & -0.22412 & 56 & 6 & 0 & -2.7907 & 0.552307 & 3.895181 \\
\hline 13 & 1 & 0 & 3.16739 & 5.46158 & -1.00879 & 57 & 1 & 0 & -2.42168 & -0.17801 & 4.609606 \\
\hline 14 & 6 & 0 & -2.82318 & 2.560787 & -1.07405 & 58 & 6 & 0 & -2.51697 & 0.368387 & 2.537424 \\
\hline 15 & 6 & 0 & 1.354425 & 0.309036 & -0.67802 & 59 & 1 & 0 & -1.93821 & -0.4999 & 2.222479 \\
\hline 16 & 1 & 0 & 1.381619 & 0.69992 & -1.70291 & 60 & 6 & 0 & 6.297311 & 0.647303 & -2.51474 \\
\hline 17 & 1 & 0 & 1.2575 & -0.78353 & -0.78693 & 61 & 1 & 0 & 7.201489 & 1.235675 & -2.64607 \\
\hline 18 & 6 & 0 & 3.150363 & 3.357044 & -0.56379 & 62 & 6 & 0 & -2.16932 & 3.708807 & -0.59198 \\
\hline 19 & 1 & 0 & 3.122681 & 3.069301 & -1.61172 & 63 & 1 & 0 & -1.66115 & 3.690454 & 0.367898 \\
\hline 20 & 6 & 0 & 5.378702 & 1.004166 & -1.52619 & 64 & 6 & 0 & -6.04334 & -1.82773 & -1.6906 \\
\hline 21 & 1 & 0 & 5.576307 & 1.869479 & -0.89997 & 65 & 1 & 0 & -6.82824 & -2.4957 & -2.03549 \\
\hline 22 & 6 & 0 & 3.218171 & 5.094898 & 1.12022 & 66 & 6 & 0 & 3.230166 & 4.124214 & 2.124115 \\
\hline 23 & 1 & 0 & 3.242393 & 6.149267 & 1.38261 & 67 & 1 & 0 & 3.259991 & 4.418496 & 3.169588 \\
\hline 24 & 6 & 0 & 3.962179 & -0.87389 & -2.1688 & 68 & 6 & 0 & -3.49695 & 3.796156 & -3.05176 \\
\hline 25 & 1 & 0 & 3.071822 & -1.48897 & -2.04263 & 69 & 1 & 0 & -4.01444 & 3.825612 & -4.00657 \\
\hline 26 & 6 & 0 & -4.7268 & -2.02552 & -2.10724 & 70 & 6 & 0 & -2.18575 & 4.889203 & -1.33659 \\
\hline 27 & 1 & 0 & -4.47282 & -2.84825 & -2.76882 & 71 & 1 & 0 & -1.68461 & 5.773706 & -0.95251 \\
\hline 28 & 6 & 0 & 6.052868 & -0.46232 & -3.32935 & 72 & 6 & 0 & -5.3461 & 0.061085 & -0.35118 \\
\hline 29 & 1 & 0 & 6.769834 & -0.73889 & -4.09796 & 73 & 1 & 0 & -5.59112 & 0.853252 & 0.351174 \\
\hline 30 & 6 & 0 & -3.70559 & -1.1824 & -1.65895 & 74 & 6 & 0 & -2.8491 & 4.934497 & -2.56675 \\
\hline 31 & 1 & 0 & -2.68975 & -1.3783 & -1.99157 & 75 & 1 & 0 & -2.86255 & 5.855575 & -3.14338 \\
\hline 32 & 6 & 0 & 3.418109 & -0.43254 & 1.406814 & 76 & 6 & 0 & -6.35393 & -0.782 & -0.81317 \\
\hline 33 & 6 & 0 & 4.685758 & -0.32293 & 2.01421 & 77 & 1 & 0 & -7.37601 & -0.63487 & -0.47493 \\
\hline 34 & 1 & 0 & 5.40586 & 0.416744 & 1.672298 & 78 & 16 & 0 & -0.94904 & -3.30374 & 1.712134 \\
\hline 35 & 6 & 0 & 5.025161 & -1.18188 & 3.055894 & 79 & 8 & 0 & -2.40948 & -3.41396 & 1.850896 \\
\hline 36 & 1 & 0 & 6.002564 & -1.09922 & 3.523958 & 80 & 8 & 0 & -0.49414 & -1.87697 & 1.597608 \\
\hline 37 & 6 & 0 & 4.115009 & -2.15816 & 3.487303 & 81 & 8 & 0 & -0.53492 & -4.02264 & 0.206166 \\
\hline 38 & 1 & 0 & 4.391183 & -2.83375 & 4.292849 & 82 & 8 & 0 & -0.11116 & -4.13245 & 2.594182 \\
\hline 39 & 6 & 0 & 2.865024 & -2.27311 & 2.88009 & 83 & 16 & 0 & 0.018614 & -3.02652 & -2.15104 \\
\hline 40 & 1 & 0 & 2.147213 & -3.03074 & 3.184406 & 84 & 8 & 0 & 1.295823 & -2.59459 & -1.51769 \\
\hline 41 & 6 & 0 & 2.510723 & -1.41008 & 1.83626 & 85 & 8 & 0 & 0.07646 & -4.31195 & -2.8482 \\
\hline 42 & 1 & 0 & 1.525991 & -1.53199 & 1.395143 & 86 & 8 & 0 & -1.1109 & -3.19881 & -0.85476 \\
\hline 43 & 6 & 0 & -3.48663 & 2.61239 & -2.31005 & 87 & 8 & 0 & -0.70521 & -1.92634 & -2.84779 \\
\hline 44 & 1 & 0 & -3.9951 & 1.73256 & -2.69248 & & & & & & \\
\hline
\end{tabular}

Note: Total energy of optimized structure II = -3589.0915928 Hartree

Table 3. Thermochemical parameters calculated by for compound II

\begin{tabular}{ll}
\hline Zero-point correction= & $\begin{array}{l}0.679505 \\
\text { (Hartree/Particle) }\end{array}$ \\
\hline Thermal correction to Energy= & 0.722073 \\
Thermal correction to Enthalpy= & 0.723018 \\
Thermal correction to Gibbs Free Energy= & 0.599403 \\
Sum of electronic and zero-point Energies= & -3586.69 \\
Sum of electronic and thermal Energies= & -3586.64 \\
Sum of electronic and thermal Enthalpies $=$ & -3586.64 \\
Sum of electronic and Thermal Free Energies $=$ & -3586.77 \\
\hline
\end{tabular}

$\mathrm{cm}^{-1}$ (Figure 8).

\subsection{General experimental procedure for oxi- dation of benzylic alcohols in water}

In a typical reaction, the alcohol as substrate $(3 \mathrm{mmol})$ was dissolved thoroughly in DM water $(5 \mathrm{~mL})$ at $40{ }^{\circ} \mathrm{C}$ and stirred well. 1,3-propanediylbis (triphenylphospho-
Table 4. Calculated frequency and intensity of some selected vibrations for compounds II

\begin{tabular}{lcc}
\hline & Frequency of vibration $\mathrm{cm}^{-1}$ & $\begin{array}{c}\text { Intensity D } \\
\left(10^{-40} \mathrm{esu}^{2} \mathrm{~cm}^{2}\right)\end{array}$ \\
\hline 1 & 3147.96 & 208.6 \\
2 & 3048.66 & 163.2 \\
3 & 2987.09 & 330.9 \\
4 & 1441.19 & 762.5 \\
5 & 1357.74 & 855.7 \\
6 & 772.81 & 1490.6 \\
\hline
\end{tabular}

nium) peroxydisulfate II, as oxidant ( $3 \mathrm{mmol}$ ) dissolved in water $(5 \mathrm{~mL})$ at $50^{\circ} \mathrm{C}$ and was added to an aqueous solution of substrate dropwise with constant stirring under reflux condition. The progress of the reaction was monitored by TLC. n-hexane/ethyl acetate (8:2) was used as mobile phase. After completion of the reaction, the mixture was cooled down, products extracted with chloroform $(3 \times 5 \mathrm{~mL})$. The crude product, thus obtained 
Table 5. Summary of Natural Population Analysis for compound II

\begin{tabular}{|c|c|c|c|c|c|c|c|c|c|c|c|c|c|}
\hline Atom & No & $\begin{array}{l}\text { Natural } \\
\text { Charge }\end{array}$ & Core & Valence & Rydberg & Total & Atom & No & $\begin{array}{l}\text { Natural } \\
\text { Charge }\end{array}$ & Core & Valence & Rydberg & Total \\
\hline $\mathrm{P}$ & 1 & 1.59568 & 9.99741 & 3.34228 & 0.06464 & 13.40432 & $\mathrm{C}$ & 45 & -0.17826 & 1.99916 & 4.16122 & 0.01788 & 6.17826 \\
\hline $\mathrm{P}$ & 2 & 1.59859 & 9.99741 & 3.34363 & 0.06037 & 13.40141 & $\mathrm{H}$ & 46 & 0.22346 & 0 & 0.77592 & 0.00063 & 0.77654 \\
\hline $\mathrm{C}$ & 3 & -0.39104 & 1.99893 & 4.36491 & 0.0272 & 6.39104 & $\mathrm{C}$ & 47 & -0.17145 & 1.99906 & 4.15464 & 0.01775 & 6.17145 \\
\hline $\mathrm{C}$ & 4 & -0.38086 & 1.99925 & 4.36303 & 0.01857 & 6.38086 & $\mathrm{H}$ & 48 & 0.23008 & 0 & 0.7693 & 0.00062 & 0.76992 \\
\hline $\mathrm{H}$ & 5 & 0.22569 & 0 & 0.77296 & 0.00135 & 0.77431 & $\mathrm{C}$ & 49 & -0.40046 & 1.99892 & 4.37423 & 0.02731 & 6.40046 \\
\hline $\mathrm{H}$ & 6 & 0.19629 & 0 & 0.80239 & 0.00132 & 0.80371 & $\mathrm{C}$ & 50 & -0.18977 & 1.99906 & 4.17303 & 0.01768 & 6.18977 \\
\hline $\mathrm{C}$ & 7 & -0.38503 & 1.99893 & 4.35693 & 0.02917 & 6.38503 & $\mathrm{H}$ & 51 & 0.21438 & 0 & 0.78494 & 0.00068 & 0.78562 \\
\hline $\mathrm{C}$ & 8 & -0.76273 & 1.99918 & 4.73785 & 0.02569 & 6.76273 & $\mathrm{C}$ & 52 & -0.20117 & 1.99915 & 4.18428 & 0.01773 & 6.20117 \\
\hline $\mathrm{H}$ & 9 & 0.32818 & 0 & 0.66959 & 0.00223 & 0.67182 & $\mathrm{H}$ & 53 & 0.21033 & 0 & 0.78906 & 0.0006 & 0.78967 \\
\hline $\mathrm{H}$ & 10 & 0.27586 & 0 & 0.72304 & 0.0011 & 0.72414 & $\mathrm{C}$ & 54 & -0.16769 & 1.99916 & 4.1512 & 0.01733 & 6.16769 \\
\hline $\mathrm{C}$ & 11 & -0.40454 & 1.99892 & 4.37644 & 0.02917 & 6.40454 & $\mathrm{H}$ & 55 & 0.21036 & 0 & 0.78904 & 0.00059 & 0.78964 \\
\hline $\mathrm{C}$ & 12 & -0.19309 & 1.99916 & 4.176 & 0.01794 & 6.19309 & $\mathrm{C}$ & 56 & -0.1795 & 1.99915 & 4.16244 & 0.0179 & 6.1795 \\
\hline $\mathrm{H}$ & 13 & 0.21442 & 0 & 0.78494 & 0.00064 & 0.78558 & $\mathrm{H}$ & 57 & 0.22305 & 0 & 0.77627 & 0.00068 & 0.77695 \\
\hline $\mathrm{C}$ & 14 & -0.38286 & 1.99892 & 4.3553 & 0.02863 & 6.38286 & $\mathrm{C}$ & 58 & -0.16798 & 1.99905 & 4.14961 & 0.01933 & 6.16798 \\
\hline $\mathrm{C}$ & 15 & -0.74161 & 1.99918 & 4.72012 & 0.02231 & 6.74161 & $\mathrm{H}$ & 59 & 0.2748 & 0 & 0.72308 & 0.00212 & 0.7252 \\
\hline $\mathrm{H}$ & 16 & 0.24465 & 0 & 0.75473 & 0.00063 & 0.75535 & $\mathrm{C}$ & 60 & -0.19753 & 1.99915 & 4.18052 & 0.01785 & 6.19753 \\
\hline $\mathrm{H}$ & 17 & 0.32692 & 0 & 0.67094 & 0.00215 & 0.67308 & $\mathrm{H}$ & 61 & 0.21055 & 0 & 0.78878 & 0.00067 & 0.78945 \\
\hline $\mathrm{C}$ & 18 & -0.179 & 1.99907 & 4.16221 & 0.01772 & 6.179 & $\mathrm{C}$ & 62 & -0.18476 & 1.99906 & 4.16798 & 0.01771 & 6.18476 \\
\hline $\mathrm{H}$ & 19 & 0.21942 & 0 & 0.7799 & 0.00069 & 0.78058 & $\mathrm{H}$ & 63 & 0.21742 & 0 & 0.78186 & 0.00072 & 0.78258 \\
\hline $\mathrm{C}$ & 20 & -0.18841 & 1.99906 & 4.17177 & 0.01759 & 6.18841 & $\mathrm{C}$ & 64 & -0.16111 & 1.99916 & 4.14473 & 0.01722 & 6.16111 \\
\hline $\mathrm{H}$ & 21 & 0.21114 & 0 & 0.78746 & 0.0014 & 0.78886 & $\mathrm{H}$ & 65 & 0.21081 & 0 & 0.7886 & 0.00059 & 0.78919 \\
\hline $\mathrm{C}$ & 22 & -0.1727 & 1.99916 & 4.15598 & 0.01756 & 6.1727 & $\mathrm{C}$ & 66 & -0.18914 & 1.99916 & 4.17231 & 0.01767 & 6.18914 \\
\hline $\mathrm{H}$ & 23 & 0.21277 & 0 & 0.78668 & 0.00055 & 0.78723 & $\mathrm{H}$ & 67 & 0.21588 & 0 & 0.78346 & 0.00065 & 0.78412 \\
\hline $\mathrm{C}$ & 24 & -0.17263 & 1.99905 & 4.15468 & 0.0189 & 6.17263 & $\mathrm{C}$ & 68 & -0.18667 & 1.99916 & 4.16993 & 0.01759 & 6.18667 \\
\hline $\mathrm{H}$ & 25 & 0.26999 & 0 & 0.72877 & 0.00123 & 0.73001 & $\mathrm{H}$ & 69 & 0.21624 & 0 & 0.78314 & 0.00062 & 0.78376 \\
\hline $\mathrm{C}$ & 26 & -0.17349 & 1.99915 & 4.15597 & 0.01836 & 6.17349 & $\mathrm{C}$ & 70 & -0.19501 & 1.99915 & 4.17799 & 0.01787 & 6.19501 \\
\hline $\mathrm{H}$ & 27 & 0.22619 & 0 & 0.77313 & 0.00068 & 0.77381 & $\mathrm{H}$ & 71 & 0.21172 & 0 & 0.78764 & 0.00063 & 0.78828 \\
\hline $\mathrm{C}$ & 28 & -0.16841 & 1.99916 & 4.15169 & 0.01755 & 6.16841 & $\mathrm{C}$ & 72 & -0.17487 & 1.99906 & 4.15863 & 0.01719 & 6.17487 \\
\hline $\mathrm{H}$ & 29 & 0.21139 & 0 & 0.78803 & 0.00058 & 0.78861 & $\mathrm{H}$ & 73 & 0.20957 & 0 & 0.78899 & 0.00143 & 0.79043 \\
\hline $\mathrm{C}$ & 30 & -0.16171 & 1.99904 & 4.14344 & 0.01922 & 6.16171 & $\mathrm{C}$ & 74 & -0.17217 & 1.99916 & 4.15558 & 0.01743 & 6.17217 \\
\hline $\mathrm{H}$ & 31 & 0.26479 & 0 & 0.73299 & 0.00222 & 0.73521 & $\mathrm{H}$ & 75 & 0.21213 & 0 & 0.78732 & 0.00055 & 0.78787 \\
\hline $\mathrm{C}$ & 32 & -0.41758 & 1.99891 & 4.39124 & 0.02743 & 6.41758 & $\mathrm{C}$ & 76 & -0.19571 & 1.99915 & 4.1789 & 0.01765 & 6.19571 \\
\hline $\mathrm{C}$ & 33 & -0.18849 & 1.99907 & 4.17188 & 0.01755 & 6.18849 & $\mathrm{H}$ & 77 & 0.21021 & 0 & 0.78914 & 0.00064 & 0.78979 \\
\hline $\mathrm{H}$ & 34 & 0.2055 & 0 & 0.79295 & 0.00155 & 0.7945 & $\mathrm{~S}$ & 78 & 2.42965 & 9.99914 & 3.34681 & 0.2244 & 13.57035 \\
\hline $\mathrm{C}$ & 35 & -0.2007 & 1.99916 & 4.18371 & 0.01784 & 6.2007 & $\mathrm{O}$ & 79 & -0.92853 & 1.9998 & 6.91788 & 0.01085 & 8.92853 \\
\hline $\mathrm{H}$ & 36 & 0.20923 & 0 & 0.79011 & 0.00066 & 0.79077 & $\mathrm{O}$ & 80 & -1.03875 & 1.9998 & 7.0216 & 0.01734 & 9.03875 \\
\hline $\mathrm{C}$ & 37 & -0.16295 & 1.99916 & 4.14666 & 0.01712 & 6.16295 & $\mathrm{O}$ & 81 & -0.45072 & 1.99989 & 6.43386 & 0.01697 & 8.45072 \\
\hline $\mathrm{H}$ & 38 & 0.21133 & 0 & 0.78808 & 0.0006 & 0.78867 & $\mathrm{O}$ & 82 & -0.93045 & 1.9998 & 6.91892 & 0.01173 & 8.93045 \\
\hline $\mathrm{C}$ & 39 & -0.17602 & 1.99914 & 4.15777 & 0.01911 & 6.17602 & $\mathrm{~S}$ & 83 & 2.43369 & 9.99913 & 3.34531 & 0.22187 & 13.56631 \\
\hline $\mathrm{H}$ & 40 & 0.25438 & 0 & 0.74456 & 0.00106 & 0.74562 & $\mathrm{O}$ & 84 & -0.99208 & 1.99979 & 6.97734 & 0.01495 & 8.99208 \\
\hline $\mathrm{C}$ & 41 & -0.15874 & 1.99904 & 4.14025 & 0.01945 & 6.15874 & $\mathrm{O}$ & 85 & -0.89976 & 1.9998 & 6.88951 & 0.01045 & 8.89976 \\
\hline $\mathrm{H}$ & 42 & 0.27064 & 0 & 0.72713 & 0.00224 & 0.72936 & $\mathrm{O}$ & 86 & -0.49887 & 1.9999 & 6.47636 & 0.0226 & 8.49887 \\
\hline $\mathrm{C}$ & 43 & -0.16663 & 1.99906 & 4.14992 & 0.01764 & 6.16663 & $\mathrm{O}$ & 87 & -0.98616 & 1.99981 & 6.9741 & 0.01225 & 8.98616 \\
\hline $\mathrm{H}$ & 44 & 0.23038 & 0 & 0.76899 & 0.00064 & 0.76962 & & & & & & & \\
\hline Total & $*$ & 0 & 133.95632 & 260.54648 & 1.4972 & 396 & & & & & & & \\
\hline
\end{tabular}

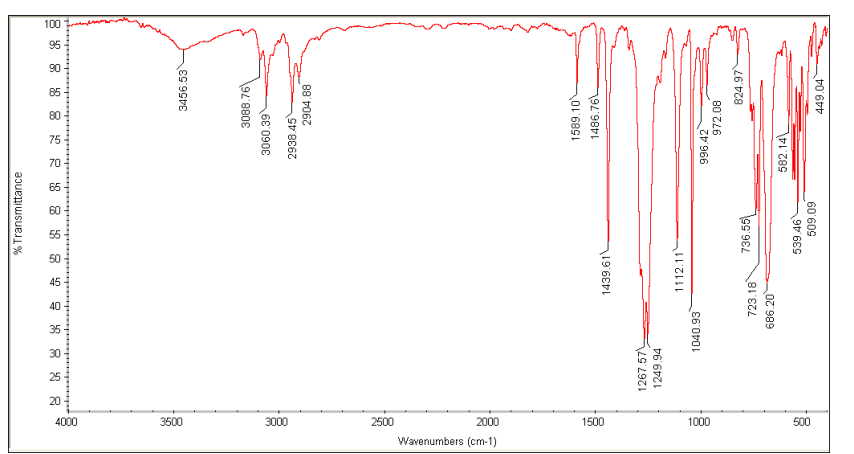

Figure 8. FT-IR spectrum of 1,3-propanediylbis (triphenylphosphonium) peroxydisulfate II (as $\mathrm{KBr}$ disk)

and then subjected to the short column of silica gel (pore size $60 \AA, 200-400$ mesh particle size) using a mixture of n-hexane and ethyl acetate (8:2) as the eluent. All of isolated products are known and physical data have been reported in literature. The main products, reaction times and isolated yields are tabulated in Table 6. Identification of products were made by comparison with their FT-IR, mass, ${ }^{1} \mathrm{H},{ }^{13} \mathrm{C}$ NMR spectra and physical data with those of authentic samples. We have reported some spectra of products as sample (see supplementary file):

4-methoxy benzaldehyde: FT-IR $(\mathrm{KBr}, \bar{v} / \mathrm{cm}): 833$ (s, aromatic C-H, bending), 1260, 1160 (s, Ar-O- $\mathrm{CH}_{3}$, stretching.), 1600, 1577 (s, C=C, stretching), 1684, 1699 (s, C=O, stretching), 2840 (s, C-H aldehyde, stretching), 2933, 2970 (C-H aliphatic, stretching), 3015, 3070 (w, $\mathrm{C}-\mathrm{H}$ aromatic, stretching). Mass Spectrum: $\mathrm{m} / \mathrm{z}$ (intensity, \% $)=136,\left(\mathrm{MeOC}_{6} \mathrm{H}_{4} \mathrm{COH}, 60\right), 107\left(\mathrm{MeOC}_{6} \mathrm{H}_{4}\right.$, 18), $92\left(\mathrm{OC}_{6} \mathrm{H}_{4}, 53\right), 77\left(\mathrm{C}_{6} \mathrm{H}_{5}, 93\right), 65\left(\mathrm{C}_{5} \mathrm{H}_{5}, 66\right), 51$ $\left(\mathrm{C}_{4} \mathrm{H}_{3}, 47\right), 39\left(\mathrm{C}_{3} \mathrm{H}_{3}, 98\right)$, and 29 ( $\left.\mathrm{CHO}, 100\right)$.

4-chlorobenzaldehyde: Mass Spectrum: $\mathrm{m} / \mathrm{z}$ (intensity, 
$\%)=140\left(\mathrm{ClC}_{6} \mathrm{H}_{4} \mathrm{COH}, 20\right), 138\left(\mathrm{ClC}_{6} \mathrm{H}_{3} \mathrm{CO}, 71\right), 110$ $\left(\mathrm{ClC}_{6} \mathrm{H}_{3}, 27\right), 75\left(\mathrm{C}_{6} \mathrm{H}_{3}, 50\right), 51\left(\mathrm{C}_{4} \mathrm{H}_{3}, 48\right)$ and $29(\mathrm{CHO}$, 100). ${ }^{1} \mathrm{H}$ NMR ( $300 \mathrm{MHz}, \mathrm{CDCl} 3, \delta$; ppm): $7.48,7.50$ (d, $\left.{ }^{3} J_{H-H}=8.1,2 \mathrm{H}\right), 7.80,7.82\left(\mathrm{~d},{ }^{3} J_{H-H}=8.1,2 \mathrm{H}\right), 9.97$ (s, $\mathrm{COH}, 1 \mathrm{H})$, ),${ }^{13} \mathrm{C}$ NMR $\left(75 \mathrm{MHz}, \mathrm{CDCl}_{3}, \delta ; \mathrm{ppm}\right.$,): 190.84, $(\mathrm{C}=\mathrm{O}), 140.88,134.72,130.89$ and $129.43(4 \mathrm{~s}$, $\mathrm{C}_{6} \mathrm{H}_{5}$ ).

Benzophenone: Mass Spectrum: $\mathrm{m} / \mathrm{z}$ (intensity, \%) $=182\left(\mathrm{C}_{6} \mathrm{H}_{5} \mathrm{CO} \mathrm{C} \mathrm{C}_{6} \mathrm{H}_{5}, 83\right), 181\left(\mathrm{C}_{6} \mathrm{H}_{5} \mathrm{CO} \mathrm{C}_{6} \mathrm{H}_{4}, 92\right)$, $105\left(\mathrm{C}_{6} \mathrm{H}_{5} \mathrm{CO}, 100\right), 77\left(\mathrm{C}_{6} \mathrm{H}_{5}, 94\right), 51\left(\mathrm{C}_{4} \mathrm{H}_{3}, 51\right)$ and 29 (CHO, 57). ${ }^{1} \mathrm{H}$ NMR (300 MHz, $\left.\mathrm{CDCl} 3, \delta ; \mathrm{ppm}\right)$ : 7.47, 7.50, $7.53\left(\mathrm{t},{ }^{3} J_{H-H}=7.5,2 \mathrm{H}\right), 7.58,7.61,7.63$ (t, $\left.{ }^{3} J_{H-H}=7.5,1 \mathrm{H}\right), 7.82,7.85\left(\mathrm{~d},{ }^{3} J_{H-H}=7.5, \mathrm{H}\right)$, ${ }^{13} \mathrm{C}$ NMR (75 MHz, $\left.\mathrm{CDCl}_{3}, \delta ; \mathrm{ppm}\right): 196.71,(\mathrm{C}=\mathrm{O})$, 137.63, 132.47, 130.08 and $128.33\left(4 \times \mathrm{s}, \mathrm{C}_{6} \mathrm{H}_{5}\right)$.

Benzil: Mass Spectrum: $\mathrm{m} / \mathrm{z}$ (intensity, $\%$ ) $=210$ $\left(\mathrm{C}_{6} \mathrm{H}_{5} \mathrm{COCO}_{6} \mathrm{H}_{5}, 4\right), 209\left(\mathrm{C}_{6} \mathrm{H}_{5} \mathrm{COCO} \mathrm{C}_{6} \mathrm{H}_{4}, 44\right), 105$ $\left(\mathrm{C}_{6} \mathrm{H}_{5} \mathrm{CO}, 100\right), 77\left(\mathrm{C}_{6} \mathrm{H}_{5}, 85\right), 51\left(\mathrm{C}_{4} \mathrm{H}_{3}, 74\right)$ and 29 (CHO, 43). ${ }^{1} \mathrm{H}$ NMR $\left(300 \mathrm{MHz}, \mathrm{CDCl}_{3}, \delta ; \mathrm{ppm}\right): 7.52$, $7.54,7.57\left(\mathrm{t},{ }^{3} J_{H-H}=7.5,2 \mathrm{H}\right), 7.66,7.69,7.71(\mathrm{t}$, $\left.{ }^{3} J_{H-H}=7.2,1 \mathrm{H}\right), 8.00,8.02\left(\mathrm{~d},{ }^{3} J_{H-H}=7.2,2 \mathrm{H}\right)$, ${ }^{13} \mathrm{C}$ NMR (75 MHz, $\mathrm{CDCl} 3, \delta$; ppm,): 194.63, $(\mathrm{C}=\mathrm{O})$, 134.94, 133.01, 129.93 and $129.06\left(4 \times \mathrm{s}, \mathrm{C}_{6} \mathrm{H}_{5}\right)$.

\subsection{Computational method}

The DFT calculations were performed by using the Gaussian 09 software package ${ }^{[63-65]}$, the B3LYP functional ${ }^{[66,67]}$ and with $6-311+\mathrm{G}^{*}$ basis set ${ }^{[68-70]}$. The population analysis has also been performed by the natural bond orbital method ${ }^{[71]}$ at $6-311+\mathrm{G}^{*}$ level of theory using the NBO program under Gaussian 2009 program package.

\section{Conclusion}

In conclusion, we describe the synthesis, thermal behavior and characterization of new quaternary phosphonium salt with oxidative anion. The specific heat capacity of the peroxydisulfate salt and related bromide salt was determined by DSC method. Furthermore, better thermal stability of title salt instead of inorganic persulfates is reported. The peroxydisulfate anion in title salt also has been used as a mild oxidizing agent for selective oxidation of benzylic alcohols in water. Ease of synthesis of II in aqueous solutions and oxidation of organic substrate by persulfate anion in water is another important aspect of phosphonium based oxidizing reagents. Also in the present paper the DFT computations and spectroscopic properties are reported.

\section{Conflicts of interest}

There are no conflicts to declare.
Table 6. Descriptive statistics and pearson correlations ( $\mathrm{N}=233)$

\begin{tabular}{|c|c|c|c|c|}
\hline Entries & Substrate & Product $^{a}$ & Time (min) & Yield $^{\mathrm{b}}(\%)$ \\
\hline 1 & & & 5 & 97 \\
\hline 2 & & & 15 & 96 \\
\hline 3 & & & 5 & 97 \\
\hline 4 & & & 15 & 97 \\
\hline 5 & & & 135 & 58 \\
\hline 6 & & & 135 & 60 \\
\hline 7 & & & 135 & 90 \\
\hline 8 & & & 90 & 93 \\
\hline 9 & & & 60 & 30 \\
\hline 10 & & & 5 & $98^{\mathrm{c}}$ \\
\hline 11 & & & 35 & 95 \\
\hline 12 & & & 60 & 40 \\
\hline 13 & & & NR & --- \\
\hline 14 & $\mathrm{OH}$ & - & NR & --- \\
\hline 15 & $\mathrm{OH}$ & --- & NR & --- \\
\hline
\end{tabular}




\section{Supporting Information}

Copies of NMR, mass, FT-IR spectra, DSC and TG/DTA thermograms, $C_{p}$ numeric data and computational study's results.

\section{Acknowledgments}

The authors are grateful for partial support of this work (grant number 3/50116) by Ferdowsi University of Mashhad Research Council. We would like to specially thank Petrochemical Research \& Technology Co to provide DSC, TG/DTG/DTA and Cp thermograms.

\section{References}

[1] Ghandi K. Green and sustainable chemistry. Green and Sustainable Chemistry, 2014, 4(1): 44-53. https://doi.org/10.4236/gsc.2014.41008

[2] Chiappe C. Ionic Liquids in Synthesis, 2008, 265-568.

[3] M. A. B. Zahoor Ullah, Zakaria Man and Amir Sada Khan ARPN Journal of Engineering and Applied Sciences,3,11,1653-1659,( 2016 )

[4] Castillo J, Coll MT, Fortuny A, et al. Cu(II) extraction using quaternary ammonium and quaternary phosphonium based ionic liquid. Hydrometallurgy, 2014, 141: 89-96. https://doi.org/10.1016/j.hydromet.2013.11.001

[5] Ma K, Li S and Weiss RG. Stereoselective Bromination Reactions Using Tridecylmethylphosphonium Tribromide in a "Stacked" Reactor. Organic letters, 2008, 10(19): 41554158 . https://doi.org/10.1021/o1801327n

[6] Esch GJ. Flame retardants: tris (2-butoxyethyl) phosphate, tris (2-ethylhexyl) phosphate, tetrakis (hydroxymethyl) phosphonium salts. World Health Organization and International Programme on Chemical Safety. 2000.

[7] Rzelewska M, Janiszewska M, Regel-Rosocka M, et al. Trihexyl(tetradecyl)phosphonium bromide as extractant for $\mathrm{Rh}(\mathrm{III}), \mathrm{Ru}(\mathrm{III})$ and $\mathrm{Pt}(\mathrm{IV})$ from chloride solutions. Chemical Papers, 2016, 70(4): 515-520. https://doi.org/10.1515/chempap-2015-0223

[8] Ovcharov VI, Okhtina OV and Golovko DA. Phosphonium Salts as Inorganic Filler Modifiers. International Polymer Science and Technology, 2002, 29(10): 17-20. https://doi.org/10.1177/0307174X0202901010

[9] Bachowska B, Kazmierczak-Baranska J, Cieslak M, et al. High Cytotoxic Activity of Phosphonium Salts and Their Complementary Selectivity towards HeLa and K562 Cancer Cells: Identification of Trinbutylnhexadecylphosphonium bromide as a Highly Potent AntiHeLa Phosphonium Salt. ChemistryOpen, 2012, 1(1): 33-38. https://doi.org/10.1002/open.201100003

[10] Xue Y, Pan Y, Xiao H, et al. Novel quaternary phosphoniumtype cationic polyacrylamide and elucidation of dualfunctional antibacterial/antiviral activity. RSC Advances, 2014, 87(4): 46887-46895. https://doi.org/10.1039/C4RA08634A
[11] Khasiyatullina NR, Vazykhova AM, Mironov VF, et al. Phosphonium salts with a dihydroxynaphthyl substituent: versatile synthesis and evaluation of antimicrobial activity. Mendeleev Communications, 2017, 27(2): 134-136. https://doi.org/10.1016/j.mencom.2017.03.008

[12] Kumar V and Malhotra SV. Study on the potential anticancer activity of phosphonium and ammonium-based ionic liquids. Bioorganic and Medicinal Chemistry Letters, 2009, 19(16): 4643-4646. https://doi.org/10.1016/j.bmcl.2009.06.086

[13] Selva M, Perosa A and No M. Phosphonium salts and Pylides. Organophosphorus Chemistry, 2016, 45: 132-169. https://doi.org/10.1039/9781782626930-00132

[14] Dey RR and Dhar SS. Synthesis and Characterization of 2-Carboxyethyltriphenyl Phosphonium Tribromide and Its Application as Catalyst in Silylation of Alcohols and Thiols Under Solvent-Free Condition. Synthetic Communications, 2014, 44(16): 2355-2363.

https://doi.org/10.1080/00397911.2014.897356

[15] Liu SY, Kumatabara Y and Shirakawa S. Chiral quaternary phosphonium salts as phase-transfer catalysts for environmentally benign asymmetric transformations. Green Chemistry, 2016, 18(2): 331-341. https://doi.org/10.1039/C5GC02692J

[16] Werner T. Phosphonium Salt Organocatalysis. Advanced synthesis and catalysis, 2009, 351(10): 1469-1481. https://doi.org/10.1002/adsc.200900211

[17] Starks CM. Phase-transfer catalysis. I. Heterogeneous reactions involving anion transfer by quaternary ammonium and phosphonium salts. Journal of the American Chemical Society, 1971, 93(1): 195-199. https://doi.org/10.1021/ja00730a033

[18] Macarie L, Simulescu V and Ilia G. PhosphoniumBased Ionic Liquids Used as Reagents or Catalysts. ChemistrySelect, 2019, 4(32): 9285-9299. https://doi.org/10.1002/slct.201901712

[19] Chaudhuri MK, Khan AT, Patel BK, et al. An environmentally benign synthesis of organic ammonium tribromides (OATB) and bromination of selected organic substrates by tetrabutylammonium tribromide (TBATB). Tetrahedron letters, 1998, 39(44): 8163-8166. https://doi.org/10.1016/S0040-4039(98)01818-8

[20] Cristiano R, Ma K, Pottanat G, et al. Tetraalkylphosphonium Trihalides. Room Temperature Ionic Liquids As Halogenation Reagents. The Journal of Organic Chemistry, 2009, 74(23): 9027-9033. https://doi.org/10.1021/jo901735h

[21] Badri R and Mostoufi A. The Synthesis and Application of 3,6-Bis(triphenylphosphonium) Cyclohexene Dichromate: An Efficient Oxidizing Agent. Phosphorus, Sulfur, and Silicon, 2006, 181(7): 1513-1519. https://doi.org/10.1080/10426500600634715

[22] Cristau HJ, Torreilles E, Morand P, et al. Bichromates de phosphonium: Reactifs d'oxydation. Tetrahedron letters, 1986, 27(16): 1775-1776. https://doi.org/10.1016/S0040-4039(00)84371-3

[23] Gholizadeh M, Ebrahimpour M, Hojati S, et al. Oxidation of benzylic and some aliphatic alcohols 1,2-ethylenebis (triphenylphosphonium) chlorochromate (EBTPPCC): As a new, an efficient and mild oxidant. Arabian Journal of Chemistry, 
2014, 7(3): 267-271.

https://doi.org/10.1016/j.arabjc.2010.10.029

[24] Tajbakhsh M, Lakouraj MM, Yadollahzadeh K, et al. Conversion of sulfides to sulfoxides and thiols to disulfides with o-xylylenebis (triphenylphosphonium tribromide). Journal of Chemical Research, 2005, 2005(12): 796-799. https://doi.org/10.3184/030823405775146988

[25] Firouzabadi H and Adibi M. Methyltriphenylphosphonium tetrahydroborate (meph3pbh4). A stable, selective and versatile reducing agent. Phosphorus, Sulfur, and Silicon and the Related Elements, 1998, 142(1): 125-147. https://doi.org/10.1080/10426509808029672

[26] Mariani A, Nuvoli D, Alzari V, et al. Phosphonium-Based Ionic Liquids as a New Class of Radical Initiators and Their Use in Gas-Free Frontal Polymerization. Macromolecules, 2008, 41(14): 5191-5196. https://doi.org/10.1021/ma800610g

[27] Dauben WG, Lorber ME and Fullerton DS. Allylic oxidation of olefins with chromium trioxide pyridine complex. The Journal of Organic Chemistry, 1969, 34(11): 35873592. https://doi.org/10.1021/jo01263a079

[28] Lou JD and Xu ZN. Selective oxidation of primary alcohols with chromium trioxide under solvent free conditions. Tetrahedron Letters, 2002, 43(35): 6095-6097. https://doi.org/10.1016/S0040-4039(02)01333-3

[29] Trahanovsky WS and Young LB. Controlled Oxidation of Organic Compounds with Cerium(IV). II. The Oxidation of Toluenes. The Journal of Organic Chemistry, 1966, 31(6): 2033-2035. https://doi.org/10.1021/jo01344a544

[30] Noureldin NA, Zhao D and Lee DG. Heterogeneous Permanganate Oxidations. 7. The Oxidation of Aliphatic Side Chains. The Journal of Organic Chemistry, 1997, 62(25): 8767-8772. https://doi.org/10.1021/jo971168e

[31] Waitkins GR and Clark CW. Selenium Dioxide: Preparation, Properties, and Use as Oxidizing Agent. Chemical Reviews, 1945, 36(3): 235-289. https://doi.org/10.1021/cr60115a001

[32] Anderson JM and Kochi JK. Silver(I)-catalyzed oxidative decarboxylation of acids by peroxydisulfate. Role of silver(II). Journal of the American Chemical Society, 1970, 92(6): 1651-1659. https://doi.org/10.1021/ja00709a039

[33] Walling C, Camaioni DM and Kim SS. Aromatic hydroxylation by peroxydisulfate. Journal of the American Chemical Society, 1978, 100(15): 4814-4818. https://doi.org/10.1021/ja00483a030

[34] Walling C and Camaioni DM. Role of silver(II) in silvercatalyzed oxidations by peroxydisulfate. The Journal of Organic Chemistry, 1978, 43(17): 3266-3271. https://doi.org/10.1021/jo00411a003

[35] Mohammadpoor-Baltork I, Hajipour AR and Haddadi R. n-Butyltriphenylphosphonium Peroxodisulfate (BunPPh3)2S2O8: an Efficient and Inexpensive Reagent for the Cleavage of CarbonNitrogen Double Bonds under Non-aqueous and Aprotic Conditions. Journal of Chemical Research, 1999, 23(2): 102-103. https://doi.org/10.1039/a806963h
[36] Perumal PT. Oxidation of Alkenes by Peroxydisulphatecopper Sulphate. Synthetic Communications, 1990, 20(9): 1353-1356.

https://doi.org/10.1080/00397919008052848

[37] Badri R, Shalbaf H and Heidary MA. 3,6-Bis (triphenylphosphonium)-cyclohexene peroxodisulfate: a highly efficient oxidant for the selective oxidation of benzylic alcohols. Synthetic Communications, 2001, 31(22): 3473-3479. https://doi.org/10.1081/SCC-100106207

[38] Shigekazu Y and Yasuyuki Y. Nickel-Catalyzed Oxidation of Allylic Alcohols with K2S2O8. Chemistry Letters, 1998, 18(8): 1361-1364. https://doi.org/10.1246/cl.1989.1361

[39] Shigekazu Y and Yasuyuki Y. Nickel-Catalyzed Dehydrogenation of Amines to Nitriles. Bulletin of the Chemical Society of Japan, 1990, 63(1): 301-303. https://doi.org/10.1246/bcsj.63.301

[40] Snook ME and Hamilton GA. Oxidation and fragmentation of some phenyl-substituted alcohols and ethers by peroxydisulfate and Fenton's reagent. Journal of the American Chemical Society, 1974, 96(3): 860-869. https://doi.org/10.1021/ja00810a035

[41] Badri R and Soleymani M. 3,6-Bis(triphenylphosphonium) cyclohexene peroxodisulfate as an efficient and mild oxidizing agent for conversion of alkylbenzenes to corresponding carbonyl compounds. Synthetic communications, 2002, 32(15): 2385-2389. https://doi.org/10.1081/SCC-120006010

[42] Badri R, Adlu M and Mohammadi MK. Synthesis and application of 1,4-bis(triphenyl phosphonium)butane peroxodisulfate for conversion of alkyl benzenes to their corresponding acylbenzenes. Arabian Journal of Chemistry, 2015, 8(1): 62-65. https://doi.org/10.1016/j.arabjc.2011.01.006

[43] Yang SG, Hwang JP, Park MY, et al. Highly efficient epoxidation of electron-deficient olefins with tetrabutylammonium peroxydisulfate. Tetrahedron, 2007, 63(24): 51845188. https://doi.org/10.1016/j.tet.2007.03.167

[44] Chen F, Wan J, Guan C, Yang J, et al. Tetrabutylammonium Peroxydisulfate in Organic Synthesis; III1. An Efficient Procedure for the Selective Oxidation of Sulfides to Sulfoxides by Tetrabutylammonium Peroxydisulfate. Synthetic communications, 1996, 26(2): 253-260. https://doi.org/10.1080/00397919608003612

[45] Park MY, Yang SG, Jadhav V, et al. Practical and regioselective brominations of aromatic compounds using tetrabutylammonium peroxydisulfate. Tetrahedron letters, 2004, 45(25): 4887-4890. https://doi.org/10.1016/j.tetlet.2004.04.112

[46] Hajipoura A, Zahmatkesha S and Ruohob A. Methyl triphenylphosphonium peroxydisulfate (MTPPP): A mild and inexpensive reagent for the cleavage of oximes, penylhydrazones and 2,4-dinitro phenylhydrazones to the corresponding carbonyl compounds under non-aqueous conditions. Journal of the Iranian Chemical Society, 2008, 5(1): S54-S58. https://doi.org/10.1007/BF03246489 
[47] Hajipour AR and Ruoho A. Methyltriphenylphosphonium peroxydisulfate and iodine as mild reagents for the iodination of activated aromatic compounds. Organic preparations and procedures international, 2005, 37(3): 279-283. https://doi.org/10.1080/00304940509354960

[48] Badri R and Soleymani M. Selective Oxidation of Benzylic Substrates to Their Corresponding Carbonyl Compounds with 3,6- Bis (Triphenylphosphonium)cyclohexene Peroxodisulfate. Synthetic communications, 2003, 33(8): 13251332 . https://doi.org/10.1081/SCC-120018692

[49] Mohammadpoor-Baltork I, Hajipour AR and Mohammadi H. Benzyltriphenylphosphonium Peroxodisulfate (PhCH2PPh3)2S2O8: a Mild and Inexpensive Reagent for Efficient Oxidation of Organic Compounds under Nonaqueous and Aprotic Conditions. Bulletin of the Chemical Society of Japan, 1998, 71(7): 1649-1653. https://doi.org/10.1246/bcsj.71.1649

[50] Fieser LF, Fieser M, Hershberg EB, et al. Carcinogenic Activity of the Cholanthrenes and of other 1:2Benzanthracene Derivatives. American Journal of Cancer, 1937, 29(2): 260268. https://doi.org/10.1158/ajc.1937.260

[51] Nokhbeh SR, Gholizadeh M, Salimi A, et al. Synthesis, crystal structure, Hirshfeld surface analysis, DFT calculations and characterization of 1,3-propanediylbis (triphenylphosphonium) monotribromide as brominating agent of double bonds and phenolic rings. Journal of Molecular Structure, 2020, 1206: 127700. https://doi.org/10.1016/j.molstruc.2020.127700

[52] Nilchi M, Ghiasi R and Mohammadi Nasab E. PseudoJahnTeller effect in Si4X4 (X=F, Cl, Br, I) molecules: a theoretical investigation. Molecular Physics, 2019, 117(5): $567-574$ https://doi.org/10.1080/00268976.2018.1527961

[53] Zandiyeh Z and Ghiasi R. A Theoretical Approach towards Identification of External Electric Field Effect on $\left(\eta{ }^{5}-\mathrm{C}_{5} \mathrm{H}_{5}\right) \mathrm{Me}_{2} \mathrm{Ta}\left(\eta{ }^{2}-\mathrm{C}_{6} \mathrm{H}_{4}\right)$. Russian Journal of Physical Chemistry A, 2019, 93(3): 482-487. https://doi.org/10.1134/S0036024419030294

[54] Nasrolahi M, Ghiasi R and Shafiee F. Stability, Electronic, and Structural Features of the Conformers of 2-Methyl1,3,2-Diheterophosphinane 2-Oxide (Heteroatom $=\mathrm{O}, \mathrm{S}$, Se): DFT and NBO Investigations. Journal of Structural Chemistry, 2019, 60(5): 746-754. https://doi.org/10.1134/S0022476619050068

[55] Hajhoseinzadeh K, Ghiasi R and Marjani A. A computational investigation on the stability and properties of the various isomers of [B7]- anion. Eurasian Chemical Communications, 2020, 2(1): 78-86. https://doi.org/10.33945/SAMI/ECC.2020.1.8

[56] Esmaeili A, Fazaeli R and Mohammadi Nasab E. Quantum chemical study of the Jahn Teller effect on the distortions of $\mathrm{XO} 2(\mathrm{X}=\mathrm{O}, \mathrm{S}, \mathrm{Se}, \mathrm{Te})$ systems. Eurasian Chemical Communications, 2020, 2(7): 739-749. https://doi.org/10.33945/SAMI/ECC.2020.7.1

[57] Ansari ES, Ghiasi R and Forghaniha A. Thermodynamic and kinetic studies of the retro-Diels-Alder reaction of 1,4 cyclohexadiene, 4H-pyran 4H-thiopyran, 1,4-dioxine, and 1,4-dithiine: a theoretical investigation. Structural Chemistry, 2019, 30(3): 877-885.

https://doi.org/10.1007/s11224-018-1241-y
[58] Ghiasi R and Amini E. Substituent and solvent effects on geometric and electronic structure of $\mathrm{C}_{5} \mathrm{H}_{5} \mathrm{Ir}\left(\mathrm{PH}_{3}\right)_{3}$ iridabenzene: A theoretical insight. Journal of Structural Chemistry, 2015, 56(8): 1483-1494. https://doi.org/10.1134/S0022476615080053

[59] Ghiasi R and Ahmadi R. The Study of Substituent Effect on Osmabenzene Complexes. International Journal of New Chemistry, 2014, 1(1): 30-40 https://doi.org/10.22034/IJNC.2014.7206

[60] Ghiasi R and Mokaram EE. Natural Bond Orbital (NBO) Population Analysis of Iridabenzene $\left(\mathrm{C}_{5} \mathrm{H}_{5} \mathrm{Ir}\right)\left(\mathrm{PH}_{3}\right)_{3}$. Journal of Applied Chemical Research, 2012, 6(1): 7-13. http://jacr.kiau.ac.ir/article_516251.html

[61] Dewar MJS. Molecular orbital theory of organic chemistry, 1969: 152-190.

[62] Carey FA and Sundberg RJ. Advanced organic chemistry: part A: structure and mechanisms, 2007.

[63] Johnson BG, Gill PM and Pople JA. The performance of a family of density functional methods. The Journal of chemical physics, 1993, 98(7): 5612-5626. https://doi.org/10.1063/1.464906

[64] Hehre WJ, Ditchfield R, Radom L, et al. Molecular orbital theory of the electronic structure of organic compounds. V. Molecular theory of bond separation. Journal of the American Chemical Society, 1970, 92(16): 1765-1771. https://doi.org/10.1021/ja00719a006

[65] Mukhina OA, Cronk WC, Kumar NNB, et al. Intramolecular Cycloadditions of Photogenerated Azaxylylenes: An Experimental and Theoretical Study. Journal of Physical Chemistry A, 2014, 118(45): 10487-10496. https://doi.org/10.1021/jp504281y

[66] Tirado-Rives J and Jorgensen JX. Performance of B3LYP Density Functional Methods for a Large Set of Organic Molecules. Journal of Chemical Theory and Computation, 2008, 4(2): 297-306 https://doi.org/10.1021/ct700248k

[67] Chen ZY and Yang ZL. The B3LYP hybrid density functional study on solids. Frontiers of Physics in China, 2006, 1(3): 339-343. https://doi.org/10.1007/s11467-006-0026-8

[68] Jensen F. Atomic orbital basis sets. Wiley Interdisciplinary Reviews: Computational Molecular Science, 2013, 3(3): 273-295. https://doi.org/10.1002/wcms.1123

[69] Davidson ER and Feller D. Basis set selection for molecular calculations. Chemical Reviews,1986, 86(4): 681-696. https://doi.org/10.1021/cr00074a002

[70] Dunning Jr TH. Gaussian basis sets for use in correlated molecular calculations. I. The atoms boron through neon and hydrogen. The Journal of chemical physics, 19892, 90(2): 1007-1023. https://doi.org/10.1063/1.456153

[71] Wolinski K, Hinton JF and Pulay P. Efficient implementation of the gauge-independent atomic orbital method for NMR chemical shift calculations. Journal of the American Chemical Society, 1990, 112(23): 8251-8260. https://doi.org/10.1021/ja00179a005 